\title{
Dewetting with conical tail formation: how to include a line friction of microscopic origin, and possibly evaporation?
}

\author{
Laurent Limat \\ Laboratoire Matière et Systèmes Complexes (MSC), UMR 7057 of CNRS and University \\ Paris Diderot, Member of USPC University Sorbonne Paris Cité, 10 rue Alice Domon et \\ Léonie Duquet, Batiment Condorcet, 75013 Paris, France.
}

laurent.limat@univ-paris-diderot.fr

\begin{abstract}
Most studies of dewetting fronts in 3D with a "corner formation", as happens behind a drop sliding down an incline are based on a generalisation of Voinov theory, with (at least implicitly) a slip length at small scale. I here first examine what happens, if instead of considering a free slip at small scale, one admits a non-zero additional line friction of microscopic origin. Concerning the selection of cone angles, I show that most features of the model are unchanged, except that the "slip length" must be replaced in the equations with an "effective" cut off that can become apparently unphysically small. I suggest that these results could explain problematical cut-offs in the hydrodynamical modelling observed recently by Winkels et al on water drops. The sole difficulty with this interpretation is the law ruling the radius of curvature of the corner tip at small scale, which remains unsatisfactory. I suggest that evaporation of the liquid should also be considered at these very small scales and propose a preliminary "toy model" to take this effect into account. The orders of magnitude are better recovered without changing the structure of the equations developed initially for "classical" wetting dynamics with silicon oil drops.
\end{abstract}

Keywords: wetting, contact line, free-surface flows, lubrication. 


\section{I - Introduction}

Despite decades of studies, wetting dynamics on a solid is still the focus of an intense activity [1-5]. In this context, one of the the perhaps simplest experiment consists in observing the shape of a drop sliding down an incline [6-12]. Several works have shown that a conical tail develops at the drop rear when the velocity is large enough $[6,7]$, the structure of both the flow [8] and the interface being well described [7-10] by an appropriate 3D generalization [13] of the "hydrodynamical model" of wetting dynamics $[14,15]$. On the other hand, depending on the liquid used, the slip cut-off assumed in this approach can be of reasonable magnitude (polymer lengths for silicon oils) [7,10] or on the contrary, completely unreasonable, i.e. extremely small for liquids such as water or liquid metals [11-12]. Understanding these effects is important for further progresses in wetting dynamics and for several applications in the field of coating, for instance immersion lithography [11]. Indeed this "corner formation" is ubiquitous and has been reported very early on liquid films extracted from a bath by a moving tape [16], as well as for air films trapped below the coating at the advancing front [17-20].

In the present paper, I suggest that these surprising apparent cut-offs could be explained by assuming some extra-dissipation at small scale [3,21,22], that could be represented by a contact line friction in the framework of combined models $[23,24]$ that mix the hydrodynamic approach with possible thermally activated jumps at small scales [21,22]. In this framework, I show that the large scale flow structure is still solely governed by the same hydrodynamical model, with exactly the same equations, but with an apparent cut-off modified by the contact line friction. This one can indeed be very small, and in appearance unphysically small, when this line friction is sufficiently large. These ideas, that I briefly suggested in a recent focus article in JFM [25] on a paper from Puttenvethil et al [12], are qualitatively compared with measurements performed on water drops by Winkels et al [11]. Most features of these experiments are recovered, and in particular the fact that the laws governing the large scale geometrical structure of the interface are unchanged, but with this very small renormalized effective cut-off. I also show that the same occurs for straight contact lines, where the same apparent cut-off can be invoked, which perhaps explains some puzzling observations of similar unphysical cut-off on water and mercury in these conditions when data are considered from a purely hydrodynamical perspective $[12,25,26]$.

However, in our 3D geometry, there is still a difficulty on the law giving the curvature at the corner tip. The singularity of the interface is limited at small scale by the same cut-off, but this one is clearly too small for what is observed, and can obviously not hold, especially as it is now the model that reaches apparently unphysical values... More technically, it appears that the exponential law involved is affected at the level of its prefactor instead of in the argument of the function, which is also inconsistent with the obtained curves. I suggest here that evaporation should perhaps be considered before the tip structure reaches these very small scales. The question of evaporation interacting with contact line dynamics is a major question in recent modelling of contact lines [28-31] trying to remove the hydrodynamic singularity, and also in the context of drying to interpret unusual aspects of "coffee-stain" effect [32-36]. I propose a tentative law including these evaporation effects, which would remain to be tested in more details on well controlled surfaces and volatile liquids, and also reconsidered with a more advanced modelling.

\section{II - Drops sliding down a plane revisited with a microscopic friction line.}

Typical pictures of a drop sliding down a plane and of its conical tail are reproduced on fig. 1, where the notations are also precised. The flow inside the tail results from a balance between viscous losses and the gradient of capillary pressure linked to the conicity. 

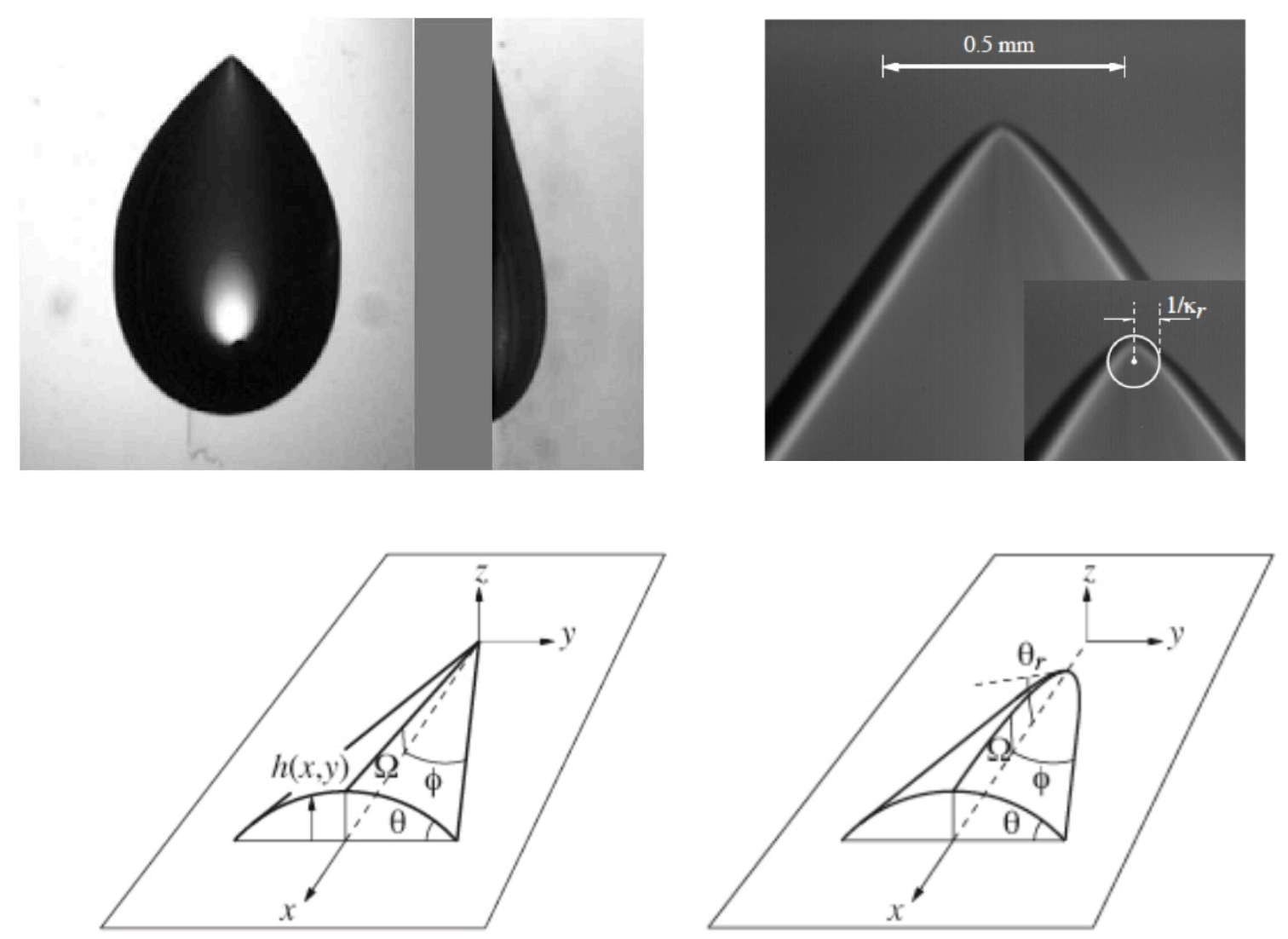

Fig. 1: (a) Top and side views of a silicon oil drop sliding down a inclined plane (from le Grand et al. [7]), under partial wetting conditions; (b) magnified vision of the cone tip at the drop rear, rounded at small scale $R=1 / \kappa_{r}$ (from Peters et al. [10]); (c) notations used here.

Assuming an approximate parabolic cross section for the interface,

$$
h(x, y) \approx x \tan \Omega\left(1-\frac{y^{2}}{x^{2} \tan ^{2} \phi}\right)
$$

together with a local balance of flow given, in the lubrication limit, by [13]

$$
V h_{x}=\frac{\gamma}{3 \eta} \nabla \cdot\left(h^{3} \nabla\left(h_{x x}+h_{y y}\right)_{x}\right)
$$

one can easily derived a relationship linking the cone angles with the capillary number $C a=$ $\eta V / \gamma$ ( $V$ drop velocity, $\eta$ liquid viscosity, $\gamma$ surface tension of the liquid). This one reads [8]

$$
\tan ^{3} \Omega=\frac{35}{16} C a \tan ^{2} \phi
$$

and is remarkably well satisfied by most of available data (see figs 2-e and 2-f in the next section). To obtain some predictions on these angles, one needs to precise the microscopic modelling of wetting at the contact line. I here propose to use Petrov "combined model" [23] (see also Appendices below), mixing both the bulk hydrodynamic dissipation with a microscopic line friction, which reads on the two inclined contact lines: 


$$
\begin{gathered}
\theta^{3}=(2 \Omega / \sin \phi)^{3}=\theta_{m}^{3}-9 C a \log (b / a) \sin \phi \\
\theta_{m}^{2}=\theta_{r}^{2}-A C a \sin \phi
\end{gathered}
$$

where $\theta_{r}$ is the static receding contact angle, $b$ a macroscopic size (typically the drop size, at which the conical tail has to match, $a$ is a small-scale cut-off, below which a line friction of microscopic origin develops, modelled by the constant $A$. This constant $A$ can be related to the more classical concept of line friction $\zeta$ used in molecular kinetic theory [38,39], where (2-b) should be usually written as:

$$
\gamma\left(\cos \theta_{r}-\cos \theta_{m}\right)=\zeta V \sin \phi
$$

which implies $A \approx 2 \zeta / \eta$ in the low angle limit. If one now considers the limit of small capillary numbers and/or slender cone of low $\phi$ value, even for "large" values of $\mathrm{A}$, these equations lead to the following equation linking $\phi$ to $C a$ :

$$
\frac{C a}{\theta_{r}^{3}}=\frac{2 \phi}{35+18 \phi^{2} \log \left(b / a^{\prime}\right)} \quad \text { with } \quad a^{\prime}=a \exp \left(-A \theta_{r} / 6\right)
$$

As one can see, these equations are the same as those developed in refs. [9-11], but in which $a^{\prime}$ plays the role of an apparent cut-off that can become extremely small in the limit of a strong microscopic slip constant $A$. The same kind of argument can be developed for the modelling of the curvature radius $R=1 / \kappa_{r}$ of the contact line at the corner tip [10], where one has to match the large scale interface slope on the symmetry axis $\Omega$ to the same modelling of the contact line at the scale of the corner tip width $R / \phi^{2}$. Roughly, one has: $\Omega^{3}=\left(\theta_{r}^{2}-A C a\right)^{3 / 2}-9 \operatorname{Ca} \log \left(2 R / a \phi^{2}\right)$, which finally yields, again in the low Ca limit:

$$
R \approx \frac{a^{\prime} \phi^{2}}{2} \exp \left(\frac{\theta_{r}^{3}}{9 C a}\right)
$$

which is the same form as the one used in [10], but with the same apparent cut-off as the one used in eq.(6). Finally, let us conclude this section with the even more simple case of a straight contact line receding normally to itself, in the vicinity of which the local slope can be written as (see more details in Appendix):

$$
\theta^{3}=\left(\theta_{r}^{2}-A C a\right)^{3 / 2}-9 \operatorname{CaLog}(b / a)
$$

In the limit of large $A$, but small enough $C a$, the first term can be developed as $\theta_{r}^{3}-(3 / 2) A C a \theta_{r}^{2}$, in such a way that (8) can be rewritten as:

$$
\theta^{3} \approx \theta_{r}^{3}-9 \operatorname{CaLog}\left(b / a^{\prime}\right)
$$

at first order upon $C a$, where $a^{\prime}$ is the effective cut-off introduced above. 

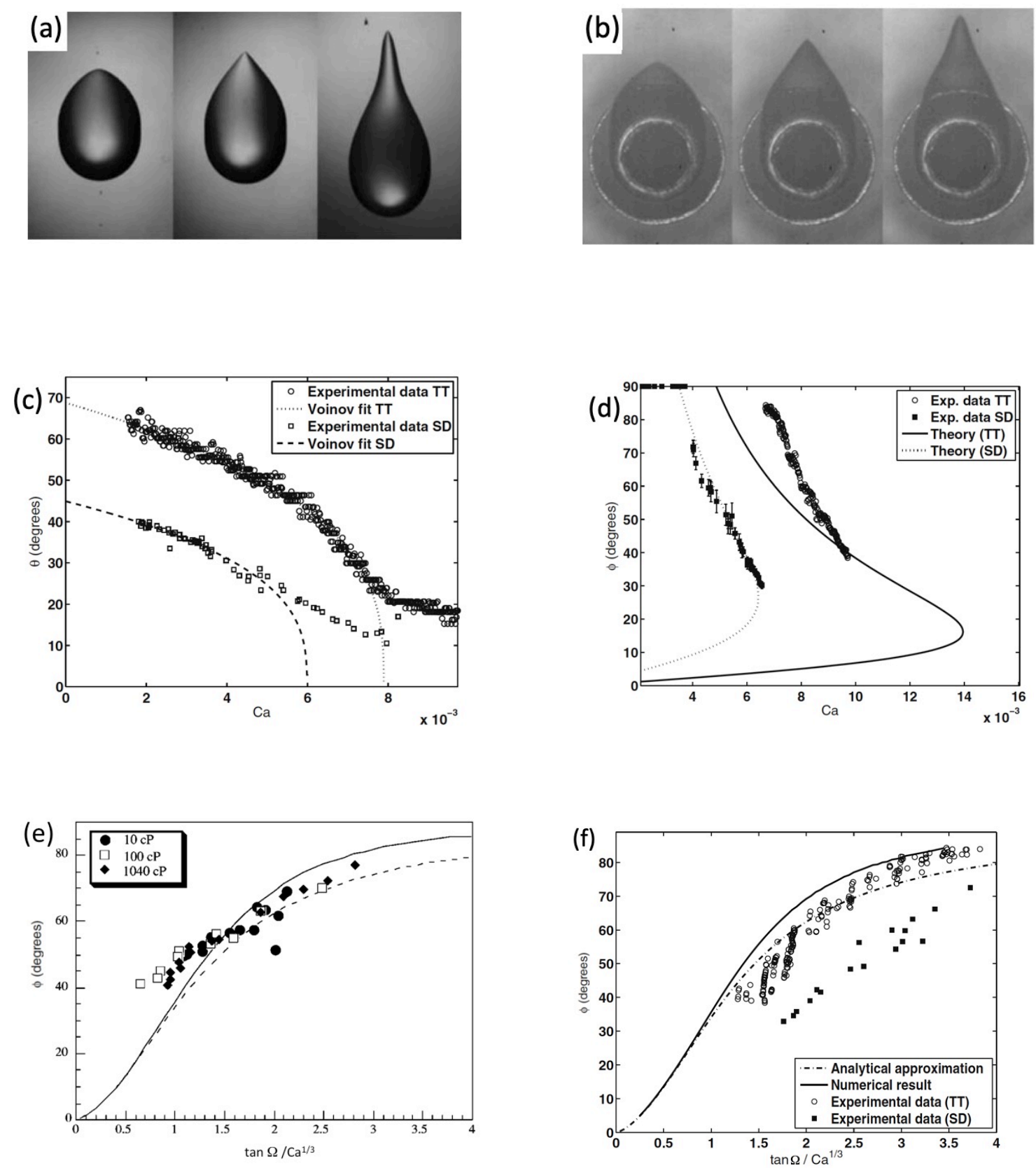

Fig. 2: (a) Typical shapes observed for silicon oil drops sliding down a plane under partial wetting conditions, Ca increasing from left to right (Peters et al [10]), (b) similar shapes observed for water drops maintained static on a glass turning table (Winkels et al. [11]); (c) dynamic contact angle observed for both experiments (SD silicon oil sliding drops, TT turning table measurements with water) at the dewetting front, fitted by eq.(9), and followed at larger Ca by the cone angle defined in a vertical plane [11]; (d) Cone angle viewed from above as a function of the capillary number for both situations, compared to the theory [11], a being replaced with a' for water (TT); (e) check of eq. (1) for silicon oils (from Snoeijer et al [8]), (f) check of eq. (1) for water (Winkels et al [11], TT). Eq.(3) corresponds to the dotted line on both figures, the continuous line being a better numerical approximate (but not analytical) obtained in refs. [8,13]. The agreement with hydrodynamics, possibly combined with a extra line friction is good, except for two things: a noticeable departure for water (TT curve) on fig. 2-d, possibly linked to the breakdown of the slender approximate for the cone, and a certain irreproducibility of data for silicon oil (second trace on fig 2-f, SD, still unexplained). 
In summary, in the three cases considered, straight contact line receding normally to itself, inclined contact line on each cone side, curved contact line at the cone apex, the large scale spatial structure should be ruled by the "hydrodynamical" model of wetting, i.e. by a balance between capillarity and viscous bulk dissipation, but with an apparent slip length that is modified by the extra friction introduced microscopically by other mechanisms. In this point of view, one should not oppose the hydrodynamical model to others (and in particular to the MKT "chemical" model of wetting), as they describe in fact complementary aspects of the same puzzle.

\section{III - Qualitative comparison with experiments: contact angle and cone angles.}

Fig. 2 compares series of experiments developed in Paris and Twente. The first series were performed by both teams on silicon oil drops, the last series being developed by Twente, alone, on water drops. Typical pictures of silicon oils drops observed for an increasing capillary number are suggested on Fig. 2-a, while Twente experiment on water drops is suggested on Fig. 2-b. In this last experiment, a water drop was maintained static in the framework of the lab, on a mobile substrate (Turning table, imitating a device of immersion lithography [37]). This was obtained by contact with a vertical tube, itself renewing the water at constant rate while compensating any loss of mass (due for instance to evaporation...). The mobile substrate was a rotating table whose surface was made up with glass covered by some (not precised) coating, providing a receding static angle of order $65^{\circ}$.

A typical record versus $\mathrm{Ca}$ of the receding angle observed from the side is reproduced on fig. 2-c, and compared to the same trace observed on a silicon oil drops. Below some critical contact angle, of order $20^{\circ}$, a conical tail appears in both case, the plots displaying then the cone angle $\Omega$, defined in a vertical plane, instead of the receding contact angle, that should now be measured in a plane normal to the contact line. Once the cone is formed, two angles are necessary to precise its geometry, $\Omega$ and $2 \phi$ the opening angle $2 \phi$ defined now in a horizontal plane. The equivalent traces for both silicon oils and water are displayed on Fig. 2$\mathrm{d}$, while the correlation between the angles $\phi$ and $\Omega$, in terms of eq. (3) are reproduced on fig. $2-\mathrm{d}$ and 2-e, respectively for silicon oils and for water.

Despite some discrepancies, it is remarkable how the whole set of data can be fitted with a hydrodynamical description. On fig.2-b, for the receding dynamical contact angle before cone formation, the data fall exactly on Voïnov prediction (eq. 9), provided that one uses appropriate cut-offs. The same occurs after cone formation for the angles $\phi$ and $\Omega$, with however some imperfections, for instance on fig. 2-d, presumably due to the fact that we are playing with low order theories (slender limit at low $\phi$ ). Note also some lack of reproducibility, in Fig. 2-f, where the data for silicon oils seem inconsistent with earlier ones reported on fig. 2-e. Anyway, in silicon oil case, the cut-off required is equal to $a=7.5 \mathrm{~nm}$ [11] when one considers a macroscopic scale $b \approx 25 \mu \mathrm{m}$, which is rather reasonable considering the typical size of the polymers (a few $\mathrm{nm}$ ). On the contrary, for water, one is constrained to use an apparent cut of very low value [11], i.e. of order $a^{\prime}=10^{-15} \mathrm{~m}(!)$, that is completely unphysical, unless one invokes the possibility suggested above in section (2): if one assumes that the nanometre remains the right scale for a microscopic cut-off (i.e. $a \approx 1$ $\mathrm{nm})$, this would require $\exp \left(-A \theta_{r} / 6\right) \approx 10^{-6}$, i.e. $A \theta_{r} \approx 36 \log (10) \approx 80$. The receding angle being of order unity when expressed in radians, this is equivalent to a line friction of order $\zeta \approx 40 \eta \approx 410^{-4} \mathrm{~Pa} s$ which is a reasonable order of magnitude for water, when compared to available literature (see for instance fig. 50 of Duvivier thesis [38]). One can thus here suggest two things: 
(1): The classical hydrodynamical model of contact line with a slip length is enough to describe straight contact lines, as well as corner properties in 3D for silicon oils.

(2): For water, it is still necessary to include hydrodynamics in the description, to describe the large scale geometry, but the microscopic picture must be modified, by replacing the "slip length" with some molecular kinetic description, that is known to work quite well for this liquid. The coexistence of both source of dissipation is well captured by the linearized version of the mixed model combining the two effects.

These conclusions are consistent with previous works of Mons team comparing different fluids of different viscosities and surface tensions (see fig. 18 in ref. [38] again, or ref. [25], showing that water experiments require absolutely to invoke some line friction in the description, not reducible to hydrodynamics), while the pure hydrodynamic modelling alone seemed enough for low surface tension liquids, such as silicon oils. Note also that despite the relatively high value found for $A / 2 \eta$, the observed behaviours can not be only interpreted with a line friction alone as attempted for instance in [40], that would lead to a saddle point $(\Omega=0)$ instead of the conical structure, separating two oblique contact lines of inclination given by Blake and Ruschak formula [16]. This is due to the physics contained in eq. (3), which has absolutely no equivalent in molecular kinetic approach of wetting dynamics.

\section{IV - The puzzle on the corner tip: should not we include evaporation and/or inertia, here?}

Another way to investigate the mechanism of corner formation consists in following the curvature at the tip for increasing capillary numbers. This study was performed for silicon oils by Peters et al [10], and generalized to water by Winkels et al [11]. Typical pictures are reproduced on Fig. 3-a where one sees clearly the progressive increase of curvature when the contact line velocity is increased.

This evolution is plotted on fig. 3-b, where one sees qualitatively a sharp growth very similar to the one predicted by eq. (7). The parameters of this growth are checked on fig. 3-c, with a Logarithmic plot of curvature upon $1 / \mathrm{Ca}$. In fact, again, only the data obtained with silicon oils are consistent with this law, with a cut-off $a^{\prime}=a$ of nanometre value. For water, there are two surprises:

- The slope of the line giving $\log \left(R_{0} / R\right)$ versus $1 / \mathrm{Ca}$ is not the right one, Winkels reporting the need to redefine the receding contact angle from its "true" value to an effective one equal to $56^{\circ}$ instead of $65^{\circ}$.

- The prefactor is much larger than the scale $a^{\prime}$ calculated in section (2), and it is not a surprise as $a^{\prime}$ is much below the molecular size, and thus cannot be a reasonable scale for a "true" length measured directly on the interface structure.

To the opinion of the writer, both effects can be understood as follows: the balance between macroscopic as well as microscopic friction and capillary pressure should lead here to a very small scale for the formed singularity at the corner tip, that seems to be able to reach levels below the nanometre (!). Obviously, some other physical mechanism will happen before $\mathrm{R}$ reaches so small values. 
a)

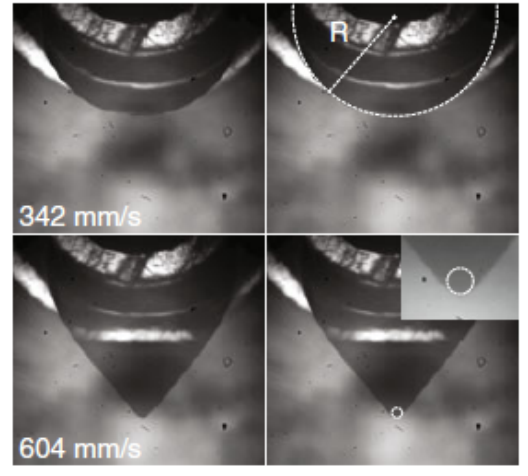

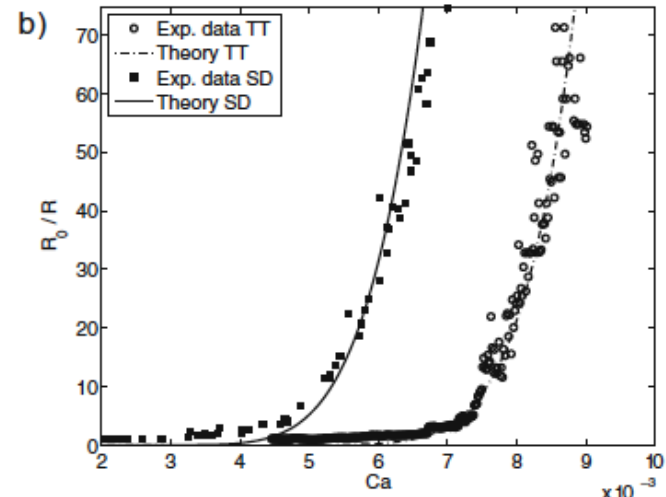

c)

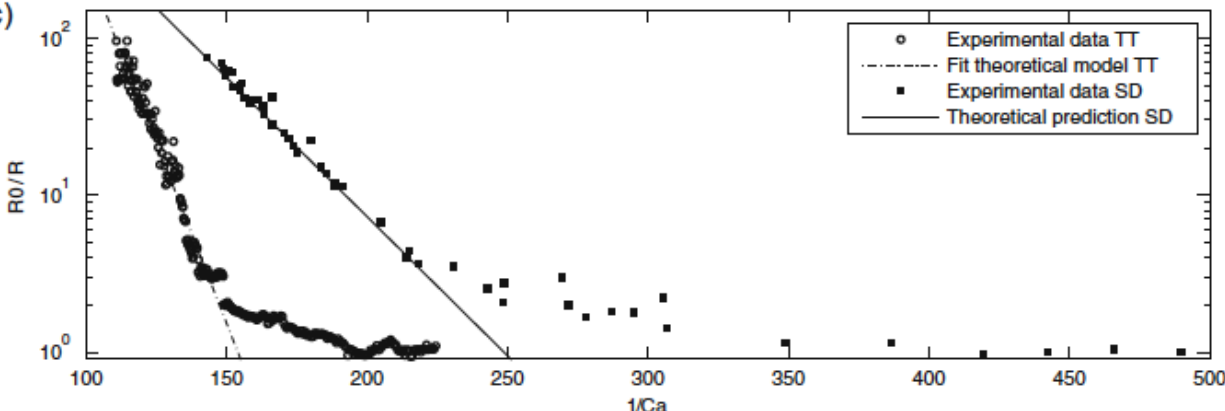

Fig. 3: (a) Pictures of the corner tip obtained for increasing capillary numbers by Twente team (Winkels et al,[11]), (b) divergence of tip curvature normalized by its initial value, (c) same as (b), to test the equivalent of eq. (7) in ref. [11] (pictures and curves extracted from Winkels et al, ref.[11])

At this step, there are two effects in which one can think: inertia of the liquid and liquid evaporation. Usually inertia is negligible in wetting problems, as the viscous stresses are diverging when one becomes closer and closer to the contact line, and dominate any residual inertia. However, recently, its effect has been invoked [41] to try to explain some departure to the $\Omega-\phi$ relation, for low viscous fluids such as water, and some surprise was also observed in problems like the pearling instability of liquid filaments [42]. Now, if we follow Kim et al. [41], the sole effect of inertia would be to add a modification of the effective pressure via a $\rho \mathrm{V}^{2}$ term. This term has no gradient in the $\mathrm{x}$-direction, or this one nearly vanishes, as the liquid must move as a whole at constant speed V. A residual effet can however be expected, if we consider 3D-effects, the flow lines being curved when reaching the contact line normally to itself $[8,43,44]$. Assuming that inertia term, in Navier Stokes equation, scales roughly as $\rho V_{\mathrm{y}} \partial \mathrm{V}_{\mathrm{x}} / \partial \mathrm{y} \approx \rho \mathrm{V}^{2} / \mathrm{R}$ while viscous effects are of order $\eta \partial^{2} \mathrm{~V}_{\mathrm{x}} / \partial_{2} \mathrm{z}^{2} \approx \eta \mathrm{V}^{2} / \mathrm{h}^{2} \approx \eta \mathrm{V}^{2} /(\Omega \mathrm{x})^{2}$, together with the geometrical relation $\mathrm{R} \approx \mathrm{x} \phi$ for a slender rounded cone, one gets that inertia should matter when the following Reynolds number is larger than 1:

$$
\operatorname{Re} \approx(x V / v)\left(\Omega^{2} / 2 \phi\right)
$$

With the orders of magnitude $\mathrm{V} \approx 0.1 \mathrm{~m} / \mathrm{s}, v \approx 10^{-5} \mathrm{~m}^{2} \mathrm{~s}^{-1}, \Omega \approx 0.3 \mathrm{rad}, \phi=0.6$ one gets that $\mathrm{Re}>1$ for only $x>1 \mathrm{~mm}$, which is a bit highn to influence what happens at the tip of the drop tail. At the scale of the rounded corner tip, i.e. $\mathrm{x} \approx 3 \mu \mathrm{m}$, Re does not exceed $310^{-3}$ which remains extremely low. There are thus very few probabilities that these effects could explain our problem of R selection. 
Another possible idea would be to imagine at very small scale some complex tip-splitting effects, whose average effect would limit the divergence (I thank one of the referees for this suggestion), but when looking to the pictures, we see a well defined curved and regular contact line at a scale of order $100 \mu \mathrm{m}$, using a camera and the required optics at a resolution of $1 \mu \mathrm{m}$. We do not see any rugosity or any tip formation between 1 and $100 \mu \mathrm{m}$.

Finally, another natural candidate is here the evaporation of the liquid, water being known as a very volatile liquid. This idea can be related to previous works performed on evaporation near contact lines by different teams. One can first mention the well known study of Deegan et al [32] who emphasize the existence of a divergence of evaporation near contact lines that scales as:

$$
J(x) \approx \frac{J_{0}}{\sqrt{x}}
$$

where $\mathrm{x}$ is the distance to contact line. This divergence is known to amplify the flow of the liquid towards the contact line induced by evaporation, which in turn amplifies the tendency to deposit solutes at a drying contact line ("coffee stain" effect). This divergence is ruled by the harmonic nature of water diffusion in air (Laplacian of concentration is equal to zero). In a vertical plane, normal to the contact line, this one becomes a $2 \mathrm{D}$ geometrical singularity around which diffusion lines are trying to escape. The mathematical description of this leads to the expression of $J_{0}$ that reads:

$$
J_{0}=\frac{D}{\sqrt{\lambda}} \frac{C_{w}^{s a t}}{\rho_{w}}
$$

in which $D$ is the diffusivity of water in air, $C_{w}{ }^{\text {sat }}$ the saturation concentration in air (supposed to hold at the free surface of water), and $\rho_{w}$ the mass density of water. The length scale $\lambda$ is a typical length that constrains the vapour flow at large scale. It can be the thickness of some boundary layer for evaporation, imposed for instance by the air flow associated to substrate motion in Twente experiment, of, for a highly curved contact line its radius of curvature $\left(R\right.$ here). In standard conditions, for water vapour in air, $D \approx 2.410^{-5} \mathrm{~m}^{2} \mathrm{~s}^{-1}$, $\rho_{W}=1000 \mathrm{~kg} / \mathrm{m}^{3}, C_{w}{ }^{\text {sat }} \approx 510^{-3} \mathrm{Kg} / \mathrm{m}^{3}$, one has $J_{0} \lambda^{1 / 2} \approx 10^{-10} \mathrm{~m}^{2} \mathrm{~s}^{-1}$.

We have perhaps here a much more convincing candidate to explain the $\mathrm{R}$ selection at small scale as we have now an effect that increases when the scale $\mathrm{x}$ goes to zero, instead of having an effect rather at large scale, when $\mathrm{x}$ increases. To get some order of magnitude, we can seek now at which scale evaporation will be able to modify appreciably the flow of the liquid. Very close to the tip, we can assume that there is only one scale that rules evaporation, i.e. that the evaporation flux towards air is of order $\mathrm{DC}_{\mathrm{w}}{ }^{\text {sat }} /\left(\rho_{\mathrm{w}} \mathrm{x}\right)$, which implies a flux of liquid leaving the cone between 0 and $x$ of order $\left(D_{w}{ }_{w}{ }^{\text {sat }} /\left(\rho_{w} x\right)\right)\left(x^{2} \phi\right) \approx\left(D C_{w}{ }^{\text {sat }} x \phi\right) / \rho_{w}$. This $1 / x$ divergence estimate is consistent with rworks from Popov and Witten, on evaporation of liquid triangular sectors [45] (apart some slight geometrical modification of the exponent -1). This must be compared to the flux linked to sliding velocity of the drop across a section located at $\mathrm{x}$, i.e. to $\mathrm{Vx}^{2} \phi \Omega$. This means that evaporation will perturb the flow when the following adimensional number is larger than 1, i.e. when

$$
\frac{D c_{w}^{\text {sat }}}{3 \rho_{w} V \Omega x}>1
$$

Again, with the available orders of magnitude, $\mathrm{D} \approx 2.410^{-5} \mathrm{~m}^{2} \mathrm{~s}^{-1}, r_{W}=1000 \mathrm{~kg} / \mathrm{m}^{3}, C_{w}{ }^{\text {sat }} \approx 510^{-3}$ $\mathrm{Kg} / \mathrm{m}^{3}, \Omega \approx 0.3 \mathrm{rad}$, evaporation should matter only for x smaller than $1 \mathrm{~nm}$... This apparently 
rather deceiving value means that the corner flow structure is not influenced by evaporation, in practice, and that we can still trust equations such as eq.(3).

However this does not mean that the radius of curvature, at the tip will remain unaffected. There is another effect that will matter: In fact, the strong evaporation flow near contact line will modify a lot the effective static and dynamic contact angle which must have some effect on the matching procedure that was used to calculate $\mathrm{R}$. This idea is at the basis of the next section.

\section{$\mathbf{V}$ - A tentative calculation of $\mathbf{R}$, in presence of liquid evaporation.}

The influence of a diverging evaporation has been only recently considered in hydrodynamical models of contact lines dynamics [28-31], and it has even been proposed that evaporation is a general phenomenon that regularizes the hydrodynamical singularity at contact lines [30]. A difficulty is here that no models are available combining hydrodynamics, evaporation and a possible line friction of molecular origin (or related to pinning/depinning events on the solid surface, as in [21]). It is however possible to develop a semi-qualitative argument by a slight heuristic generalization of the expression of dynamic contact angle proposed by Berteloot et al [33] for a receding evaporating contact line:

$$
\theta_{d}^{3} \approx \theta_{m}^{3}-9 \operatorname{CaLog}\left(\frac{x}{a}\right)+24 \frac{\eta J_{0}}{\gamma \theta_{m}}\left(\frac{1}{a^{1 / 2}}-\frac{1}{x^{1 / 2}}\right)
$$

in which we have replaced the static, macroscopic, receding angle by the one assumed in molecular kinetic theory (eq. 4-b), assuming also that all the angles here are small and slowly varying with $x$ (which is not really the case here...). Using exactly the same argument as in ref. [10-11], i.e. that $\theta_{d} \approx \Omega$ at a distance of order $x \approx 2 R / \phi^{2}>>a$ of contact line (matching condition between the cone and its rounded tip), one finally yields the obtained value for the tip radius:

$$
\begin{aligned}
& R \approx \frac{a^{\prime} \phi^{2}}{2} \exp \left[\frac{\theta_{r}^{3}}{9 C a}\left(1+24 \frac{\eta J_{0}}{\gamma a^{1 / 2} \theta_{r}^{4}}\right)\right] \\
& a^{\prime} \approx a \exp \left[-\frac{A \theta_{r}}{6}\left(1-12 \frac{\eta J_{0}}{\gamma a^{1 / 2} \theta_{r}^{2}}\right)\right]
\end{aligned}
$$

As one can see, now, the decrease of a' scale will be limited by evaporation. At low $\mathrm{Ca}$, we can expect that the spatial scale $\lambda$ that rules $J_{o}$ will be some constant thickness (or at least slowly varying with $\mathrm{Ca}$ ) of a boundary layer for evaporation near the glass plate, and at higher capillary number, we can imagine that $\lambda$ will progressively adjust itself to $R$, which implies that eqs. (15) will prevent $R$ to reach very low values. Not also that these ideas are perhaps consistent with the two regimes observed on the curve $\log (R o / R)$ versus $1 / C a$ observed on fig. (3-c), that could correspond to the two different regimes of evaporation for $\lambda$. An interesting feature is also that we recover in (15-a) that the argument of the exponential function is modified, which will lead to an apparent contact angle $\theta_{r}$ that will differ from the expected one, as observed by Winkels, but unfortunately the sign of the effect is reversed, the effective angle being larger (and not smaller) than the macroscopic one... A more refined analysis would be here required, as eq.(14) is just a "toy model", imagined for very small contact angles, that also needs improvements, and whose predictions must be taken with 
cautions. We now just limit ourselves to some calculations of orders of magnitude, in order to see if our idea to invoke evaporation is reasonable or not.

For a static drop of millimetre size, with low contact angles, in standard conditions, the orders of magnitude reminded above lead to $J_{o} \approx 510^{-9} \mathrm{~m}^{3 / 2} \mathrm{~s}^{-1}$ [32,33]. In addition, this quantity scale as $R^{-1 / 2}$, where $R$ is the radius of the drop, which equivalent is here the radius of curvature $R$ of the corner tip, itself of order 10 to $100 \mu \mathrm{m}$ [11], which implies here a larger effective value $J_{o} \approx 510^{-8} \mathrm{~m}^{3 / 2} \mathrm{~s}^{-1}$. With typical viscosity and surface tension values for water of order $\eta=10^{-3} \mathrm{Pas}$, and $\gamma=80 \mathrm{mN} / \mathrm{m}$, and speculating on a microscopic scale $a$, of molecular size , the typical correction in (10) is of order : $24 \eta J_{o} /\left(\gamma a^{1 / 2}\right) \approx a$ few $10^{-3}$ which is just too small to have a measurable effect here. Now, we have to take care on the angle dependencies, this factor being divided in (15-a) and (15-b), respectively by $\theta_{r}{ }^{2}$ and $\theta_{r}{ }^{4} \ldots$ which are here unfortunately of order unity (!). This may seem again a bit deceiving, but we have also to remind that we are here dealing with theories valid for very low interface slopes, extrapolated to larger values, and in addition slowly varying with the distance to the contact line. All these approximates allow us to develop something analytical, but are clearly not valid here.

In such conditions, one would have to generalize the modelling to a non-linear high angle situation, which is difficult, or to focus on linearized theories in a low range of capillary numbers. In such a situation, it is not obvious that the macroscopic receding angle is the relevant reference angle here, on the new branch of solutions that appear at cone onset, and not for instance a fraction of this angle or even some other reference angle. One can think for instance to the cone angle $\Omega$ at cone onset, for which, in addition, there is still no model available. For all these reasons, one can question globally this modelling that is very rough and suggests that other typical angles could be involved in a "true" theory. For instance, if one replaces $\theta_{r}$ by something more relevant to the cone structure, for instance $\Omega \approx 0.1 \mathrm{rad}$ at cone onset, one gets some effective parameters $12 \eta J_{o} /\left(\gamma a^{1 / 2} \Omega^{2}\right)$ and $24 \eta J_{o} /\left(\gamma a^{1 / 2} \Omega^{4}\right)$ of order 1 and 10 , both having orders of magnitude able to perturb eq. (15). So, though the present picture is at first look not completely satisfactory, I think that evaporation should indeed be considered in this question of corner tip radius selection with water, but presumably with a more advanced description that the present one. Kelvin effect linked to the strong curvature of the interface should be included, for instance, as in refs. [28-29].

\section{V - Conclusion}

In short, I have examined the replacement of the classical no-slip condition at contact line by some line friction inspired by the molecular kinetic theory on the "corner formation" by dewetting in 3D, as observed at the rear of sliding drops of water. This approach, in terms of the "combined model" imagined by Petrov and Petrov seems able to explain why the same laws could hold as for the first experiments with silicon oils, but with apparently unphysical spatial cut-off of very low values. Now it fails to describe what happens with the selection of the contact line curvature at the tip of the corner. I have suggested that liquid evaporation should be included in the description to get a more reasonable description and provided a "toy model" to do this. Again, this approach preserves the structure of the equations found initially when considering silicon oil drops, and improves the problem with orders of magnitude for the cut off, but the predicted influence of the reference static receding contact angle remains problematical.

Further works are necessary to get a complete understanding, with new and better controlled experiments (substrate properties, liquid volatility, etc.), as well as new and more refined approaches of wetting dynamics, especially with respect to the consistency of $\mathrm{Ca}$ developments, or to the physical basis of a line friction of microscopic origin, and also with 
respect to evporation [28-31, 46, 47]. A question that remains asked is also what happens for large static contact angles, the theory developed here holding only in the limit of low interface slopes. Though observations of a conical structure has been reported, experimentally in such conditions [12], recent numerical simulations by molecular dynamics are unable to reproduce it in the same limit [44], despite a well defined transition to pearling very similar to experiments. To conclude, I mention that this idea to combine a line friction with standard hydrodymical models to interpret dewetting data from immersion lithography has been first proposed independently during the coating symposium ECS2013 in Mons by M. Riepen [37].

Acknowledgments: I thank T. Blake, P. Colinet, C. Colosqui, A. Daerr, M. Riepen, M. Roché, J. Snoeijer, K. Winkels, for exchanges and discussions. This paper has also greatly benefited from a stay in University of Mons, and discussions with J. de Coninck.

\section{References}

1. P. G. de Gennes, Rev. Mod. Phys. 57, 827 (1985)

2. T.D. Blake and K.J. Ruschak, in "Liquid film coating", edited by P.M. Schweizer and S.F. Kistler, Chapman and Hall, London 63-97 (1997)

3. J. De Coninck and T. D. Blake, Ann. Rev. of Mat. Research, 38, 1-22 (2008)

4. D. Bonn, J. Eggers, J. Indekeu, J. Meunier and E. Rolley, Rev. Mod. Phys. 81, 739-805 (2009)

5. J.H. Snoeijer and B. Andreotti, Ann. Rev. Fluid Mech. 45, 269 (2013)

6. T. Podgorski, J. M. Flesselles and L. Limat, Phys. Rev. Lett. 87, 036102-036105 (2001)

7. N. Le Grand, A. Daerr and L. Limat, J. Fluid Mech. 541, 293-315 (2005)

8. J. H. Snoeijer, E. Rio, N. Le Grand and L. Limat, Phys. Fluids 17, 072101 (2005)

9. J. H. Snoeijer, N. Le Grand, L. Limat and H. A. Stone, J. Eggers, Phys. Fluids 19, 042104 (2007)

10. I. Peters, J. H. Snoeijer, A. Daerr and L. Limat, Phys. Rev. Lett. 103,114501 (2009)

11. K. G. Winkels, I. R. Peters, J. Snoeijer, F. Evangelista, M. Riepen, A. Daerr and L. Limat, Eur. Phys. J. Spec. Topics. 192, 195-205 (2011)

12. B. A. Puthenveettil, V. K. Kumar and E. J. Hopfinger, J. Fluid Mech. 726, 2013, 26-61 (2013)

13. L. Limat and H. A. Stone, Europhys. Lett. 65 (3), pp. 365-371 (2004)

14. O. V. Voinov, Fluid Dyn. 11, 714 (1976)

15. R. G. Cox, J. Fl. Mech. 168, 169-195 (1986)

16. T. D. Blake and K. J. Ruschak, Nature 282, 489 - 491 (1979)

17. R. Burley and B. S. Kennedy, Chem. Engn. Sci. 31, 901 (1976)

18. H. Benkreira and J. B. Ikin, Chem. Engn. Sci. 65, 1790-1796 (2010)

19. E. Vandre, M. S. Carvalho and S. Kumar, Physics of Fluids 25, 102103 (2013)

20. M. He and S. R. Nagel, Phys. Rev. Lett. 122, 018001 (2019)

21. T.D. Blake and J.M. Haynes, J. Coll. Interface Sc., 30, 421-423 (1969)

22. E. Rolley and C. Guthmann, Phys. Rev. Lett. 98, 166105 (2007); H. Perrin, R. Lhermerout, K. Davitt, E. Rolley, and B. Andreotti, Soft Matter 14, 1581-1595 (2018)

23. P. G. Petrov and J. G. Petrov, Langmuir 8, 1762-1767 (1992)

24. M. de Ruijter, M. Charlot, M. Voué and J. De Coninck, Langmuir, 16 2363-2368 (2000)

25. L. Limat, J. Fl. Mech., 738, 1-4 (2014)

26. M. Fermigier and P. Jenffer, J. of Coll. and Interface Sc., 146, 226-241 (1991)

27. P. Hayoun, "Partial wetting of thin liquid films in polymer tubes", Thesis, University Paris 6 (2016).

28. A. Rednikov and P. Colinet, Phys. Rev. E, 87, 010401(R) (2013)

29. V. Janecek, B. Andreotti, D. Prazak, T. Barta, and V. S. Nikolayev, Phys. Rev. E, 88, 060404(R) (2013)

30. Y. Pomeau, C. R. Acad. Sci., Ser. IIb 328, 411 (2000)

31. L. M. Pismen and Y. Pomeau, Phys. Rev. E 62, 2480 (2000) 
32. R. D. Deegan, O. Bakajin, T. F. Dupont, G. Huber, S. R. Nagel and T. A. Witten, Nature 389, 827-829 (1997)

33. G. Berteloot, C.-T. Pham, A. Daerr, F. Lequeux and L. Limat, Europhys. Lett. 8314003 (2008)

34. C.-T. Pham, G. Berteloot, F. Lequeux and L. Limat, Europhys. Lett. 93, 69901 (2010)

35. J. Eggers and L. M. Pismen, Phys. Fluids 22, 112101229 (2010)

36. F. Doumenc and B. Guerrier, Eur. Phys. J. Spec. Top. 197, 281 (2011)

37. M. Riepen, "Dynamics of dewetting in immersion lithography", extended abstract from ECS 2013, European Coating Symposium (2013), edited by J. De Coninck; see also in the same booklet, L. Limat, "Straight contact lines on soft solids and broken contact lines on hard substrates".

38. D. Duvivier, "Comportement de gouttes de mélange en situation de mouillage extrème", Thèse de Doctorat de l'Université de Mons, Presses Universitaires de Mons (2013), ISBN 978-2-87325-076-8

39. D. Duvivier, T. Blake, and J. De Coninck, Langmuir 29, 32, 10132-10140 (2013)

40. M. Ben Amar, J. L. Cummins and Y. Pomeau, Phys. Fluids 15, 2949-2960 (2003)

41. H. Kim, C. Poelma, G. Ooms and J. Westerweel, J. Fl. Mech. 762, 393-416 (2015)

42. J. R. Castrejón-Pita, A. A. Castrejón-Pita, S. S. Thete, K. Sambath, I. M. Hutchings, J. Hinch, J. R. Lister, and O. A. Basaran, Proc. Nat. Acad. Sc. 112, 15, $4582-4587$ (2015)

43. E. Rio, A. Daerr, B. Andreotti, and L. Limat, Phys. Rev. Lett. 94, 024503 (2005)

44. J. C. Fernandez-Toledano, T. D. Blake, L. Limat and J. de Coninck, J. Coll. Interface Sc., 548, 66-76 (2018)

45. Y. O. Popov and T. Witten, Phys. Rev. E, 68, 036306 (2003)

46. C. E. Colosqui, "Thermodynamics, Dynamics, and Kinetics of Nanostructured Fluid-Solid Interfaces", arXiv:1707.04750 (2017)

47. D. Sibley, A. Nold and S. Kalliadasis, J. Fluid Mech., 764, 445-462 (2015).

48. J. Eggers, Phys. Fluids, 16, 3491-3494 (2004).

\section{Appendix A.}

\section{More general formula for the tip curvature.}

Strictly speaking, eq. (7) and, consequently eq. (15), is a bit more complex that what we present here. There is an additional, weak dependence upon the $\phi$ angle, that was yet omitted in refs. (10-11), and that reads as follows:

$$
R \approx \frac{a^{\prime} \phi^{2}}{2} \exp \left[\frac{\theta_{r}^{3}}{9 C a}\right] \exp \left[-\frac{35}{144} \phi^{2}\right]
$$

This factor should also be taken into account in eq. (13) that reads in fact:

$$
R \approx \frac{a \phi^{2}}{2} \exp \left[-\frac{A \theta_{r}}{6}\left(1-12 \frac{\eta J_{0}}{\gamma a^{1 / 2} \theta_{r}^{2}}\right)\right] \exp \left[\frac{\theta_{r}^{3}}{9 C a}\left(1+24 \frac{\eta J_{0}}{\gamma a^{1 / 2} \theta_{r}^{4}}\right)\right] \exp \left[-\frac{35}{144} \phi^{2}\right]
$$

This week dependence is negligible compared to the strength of the $\exp \left[\theta_{r}^{3} /(9 \mathrm{Ca})\right]$ diverging central term, when $C a$ is progressively reduced, but should be taken into account in a more advanced modelling. 


\section{Appendix B.}

\section{Reminding Voinov approach for a single receding contact line with a slip length.}

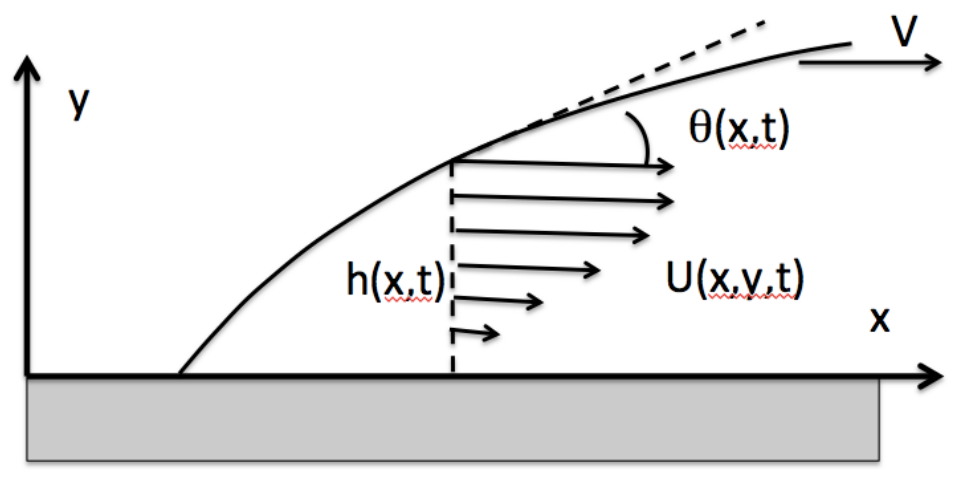

Let us consider, as on the figure, a receding contact line in situation of partial wetting, the liquid thickness being called $h(x, t)$ and the fluid velocity component, parallel to the substrate, $U(x, y, t)$. Mass conservation imposes that:

$$
\frac{\partial h}{\partial t}+\frac{\partial}{\partial x}[h<U(x, t)>]=0
$$

where $\langle U\rangle$ is the mean velocity integrated over the thickness $h$. Seeking for a solution propagating at uniform velocity, i.e. $h=h(x-V t)$, with $\partial h / \partial t=-V \partial h / \partial x$, one will find easily that the quantity $(V-U>) h$ is constant and uniform, and equal to zero if there exists a point at which $h$ goes to zero (contact line). Thus, in the lubrication approximation, one has everywhere

$$
<U>=V=-\frac{h^{2}}{3 \eta} \frac{\partial}{\partial x}\left(-\gamma h_{x x}\right)
$$

where the term $\gamma h_{x x}$ represents the capillary pressure excess linked to curvature, whose gradient drives the fluid motion. This leads to the well-known equation:

$$
h_{x x x}=\frac{3 C a}{h^{2}}
$$

in which $C a$ designates the capillary number, i.e. $C a=\eta V / \gamma$. Instead of seeking for $h(x)$, it is simpler to seek, following Voïnov, for a first integral [14], i. e. to seek for $\theta \approx h_{x}=f(h)$ where $\theta$ is the local slope of the profile, which, after noting that $\partial / \partial x=\theta \partial / \partial h$, leads to:

$$
\frac{3 C a}{h^{2}}=\theta \frac{\partial}{\partial h}\left(\theta \frac{\partial}{\partial h} \theta\right) \approx \frac{\partial}{\partial h}\left(\theta^{2} \frac{\partial}{\partial h} \theta\right)
$$

where the second equation holds for $\mathrm{Ca}$ not too large. This finally leads to a Logarithmic solution, when the curvature at large-scale $\partial \theta / \partial \mathrm{x}$ is supposed to be negligible:

$$
\theta^{3}(h)=\theta^{3}(a)-9 \operatorname{CaLog}(h / a)
$$


If we select now the reference scale a, as a very small thickness below which some free slip will occur, one can consider that viscous stresses will be inefficient to affect the contact angle selection at very small scale. $\theta(a)$ will reduce to some static equilibrium value i. e. $\theta(a)=\theta_{e}$ that , for simplicity, is identified to the static receding contact angle on fig. 2-c. In fact, it is possible to be more rigorous with the slip length concept. If one assumes a Navier boundary condition on the solid instead of $U(y=0)=0$ :

$$
\lambda \frac{\partial U}{\partial y}=U \quad \text { for } \mathrm{y}=0
$$

it is easy to show that eqs. (B-4) will reduce to:

$$
\frac{\partial}{\partial h}\left(\theta^{2} \frac{\partial}{\partial h} \theta\right) \approx \theta \frac{\partial}{\partial h}\left(\theta \frac{\partial}{\partial h} \theta\right)=\frac{3 C a}{h^{2}+3 \lambda h}
$$

The same process of integration as above gives now:

$$
\theta^{3}(h)=\theta_{e}^{3}+9 C a\left[\log \left(1+\frac{h}{3 \lambda}\right)+\frac{h}{3 \lambda} \log \left(1+\frac{3 \lambda}{h}\right)\right]
$$

In the limit $h>>\lambda$, this expression reduces to:

$$
\theta^{3}(h)=\theta_{e}^{3}-9 \operatorname{CaLog}\left(\frac{h e}{3 \lambda}\right)
$$

in which $e \approx 2.732 \ldots$ is the well-known exponential constant. This expression is completely consistent with eq. B-7, with $a=3 \lambda e$ and allows us to establish some bridge more recent versions of Voïnov description [4, 48]. Rather close to the contact line, one can assume that $h \approx x \theta_{e}$, which allows us to have an approximate expression for the $x$-dependence of $\theta$ :

$$
\theta^{3}(x) \approx \theta_{e}^{3}-9 \operatorname{CaLog}\left(\frac{e}{3 \lambda} x \theta_{e}\right)
$$

which exactly coincides with Eggers formula [48] at leading order upon $C a$. Note that the advancing case can be deduced from above by changing $V$ into $-V$, which gives here:

$$
\theta^{3}(x) \approx \theta_{e}^{3}+9 \operatorname{CaLog}\left(\frac{e}{3 \lambda} x \theta_{e}\right)
$$

More generally, in all the above formula $-\mathrm{Ca}$ can be replaced with $+\mathrm{Ca}$, while one replaces also when necessary, the receding static angle $\theta_{r}$ with the advancing static angle $\theta_{a}$. Finally all this shows that this approach leads to an apparent contact angle at a macroscopic scale $b$ given by:

$$
\theta^{3}(b) \approx \theta_{e}^{3} \pm 9 \operatorname{Ca} \log (b / a)
$$


where $b$ is the macroscopic scale considered and $a$ is some microscopic scale below which slip occurs, both being defined in the y-direction, rather.

\section{Appendix C.}

\section{Introduction of an extra line friction in Voïnov approach: towards "combined models".}

The point of view developed in Apendix B is not the sole possible, even from a hydrodynamical perspective. Instead of considering a "free slip" at small scale, one can imagine that viscous stresses are on the contrary replaced by a strong, non zero friction of microscopic origin, linked to some mechanisms that can be very diverse. It can be adsorption/desorption of molecules on the solid [2-3, 21, 38-39], or pinning/depinning of the contact line by very small defects of the solid surface $[4,22]$. The simplest idea, in this case, is to introduce some line friction $\zeta$ representative of these mechanisms, the local contact angle at very small-scale $\theta_{\mathrm{m}}$ resulting from a balance between capillarity and friction:

$$
\gamma\left(\cos \theta_{m}-\cos \theta_{r}\right) \approx(\gamma / 2)\left(\theta_{r}^{2}-\theta_{m}^{2}\right)=\zeta V
$$

This line friction can be calculated in the framework of molecular kinetic models [21], which finally leads to a value proportional to the fluid viscosity [38-39], with some prefactor depending upon the adhesion parameter, but the concept is here more general. One can assume very generally a line friction representative of microscopic effects, even without a precise knowledge of these ones, and develop calculations on this sole basis [40].

To reconnect with Voinov model, one can assume that large scale hydrodynamics have to match with his microscopic situation at some microscopic scale called again " $a$ ", which finally yields:

$$
\begin{aligned}
& \theta^{3}(b)=\theta_{m}^{3}-9 \operatorname{CaLog}(b / a) \\
& \theta_{m}^{2}=\theta_{r}^{2}-A C a
\end{aligned}
$$

with $A=2 \zeta / \eta$ as used in (4-a) and (4-b) in a direction perpendicular to the contact line. This idea is the point of departure of what is often called "combined models" of wetting dynamics as initially proposed by Petrov and Petrov [23], that we tried to use in the present paper for the cone structure formation in dewetting experiments. 